\title{
Molecular diagnostic analysis for Huntington's disease: a prospective evaluation
}

\author{
J C MacMillan, P Davies, P S Harper
}

\begin{abstract}
The availability of mutation analysis for the CAG repeat expansion associated with Huntington's disease has prompted clinicians in various specialties to request testing of samples from patients displaying clinical features that might be attributable to Huntington's disease. A series of 38 cases presenting with clinical features thought possibly to be due to Huntington's disease were analysed prospectively. In $53 \%$ of such cases presenting initially with chorea and $62.5 \%$ with psychiatric symptoms an expansion was identified, a considerable lower proportion than found in previous series where the diagnosis was considered definite on clinical and genetic grounds. Mutation analysis is likely to be of considerable value in the diagnosis of Huntington's disease, especially where the family history in previous generations is inadequate or apparently negative.
\end{abstract}

$(\mathcal{F}$ Neurol Neurosurg Psychiatry 1995;58:496-498)

Keywords: Huntington's disease; molecular diagnostic analysis

Huntington's disease is an inherited progressive neurodegenerative disease, typically with onset of choreiform movements and cognitive decline in midlife or later. ${ }^{1}$ Over $99 \%$ of patients with a firm clinical diagnosis of Huntington's disease have been shown to have an expansion in an unstable CAG trinucleotide repeat in the IT15 gene. ${ }^{2-4}$ These patients were mainly studied for research purposes and their DNA stored and subsequently analysed by units engaged in the cloning of the Huntington's disease gene. They are therefore biased in that the samples come from patients with "typical" clinical manifestations and, in the great majority, a positive family history for Huntington's disease or a disorder diagnosed as such. There have been some data published on the presence of the Huntington's disease CAG expansion in retrospective series of "sporadic" cases of Huntington's disease" namely, those without a positive family history but with a suggestive clinical phenotype, those with "suspected" Huntington's disease,${ }^{6}$ in series of patients with schizophrenia, ${ }^{7}$ and most recently in symptomatic patients reported along with results from those requesting presymptomatic prediction. ${ }^{8}$

We have found that the availability of a sensitive and specific molecular test for this genetic disorder now leads to requests for such an analysis in a wider range of clinical diagnostic situations than previously resulted in a request for DNA banking for future analysis. We here report the series of requests for Huntington's disease diagnostic mutation analysis that followed its establishment in the service laboratory of our unit.

\section{Materials and methods}

Requests for Huntington's disease mutation analysis were received from clinicians throughout England and Wales. Mutation analysis was only undertaken when the clinician had obtained informed consent from the patient (or their family where the patient was unable to give consent) and had completed a standardised data sheet detailing clinical manifestations, family history, and the results of neurological investigations such as CT and MRI. Diagnostic mutation analysis was only offered in the context of an affected patient with symptoms considered likely to be due to Huntington's disease, not in asymptomatic patients at risk who were assessed with previously validated counselling protocols, ${ }^{9}$ and for whom data are not reported here.

Blood samples $(20 \mathrm{ml})$ in EDTA were forwarded by first class post to our unit and DNA was extracted from peripheral blood leucocytes by standard techniques. Polymerase chain reaction amplification of the region of IT15 containing the CAG repeat polymorphism was carried out as described previously. ${ }^{3}$ The normal range for our laboratory, based on 1160 normal chromosomes, is nine to 34 repeats with a mean of 18 .

\section{Results}

Initial diagnostic enquiries concerning 43 cases were received over a 12 month period. Thirty nine blood samples were subsequently received for analysis from which $38(97 \cdot 4 \%)$ mutation results were successfully generated. Seventeen referrals (15 subsequent samples 
Clinical and molecular data for the cases in the series

\begin{tabular}{|c|c|c|c|c|c|}
\hline Case & Sex & $\begin{array}{l}\text { Age at } \\
\text { presentation }\end{array}$ & Presentation & $\begin{array}{l}\text { Family } \\
\text { history }\end{array}$ & Repeats \\
\hline $\begin{array}{l}1 \\
2 \\
3 \\
4 \\
5 \\
6 \\
7 \\
8 \\
9 \\
10 \\
11 \\
12 \\
13 \\
14 \\
15 \\
16 \\
17 \\
18 \\
19 \\
20 \\
21 \\
22 \\
23 \\
24 \\
25 \\
26 \\
27 \\
28 \\
29 \\
30 \\
31 \\
32 \\
33 \\
34 \\
35 \\
36 \\
37 \\
38\end{array}$ & $\begin{array}{l}F \\
M \\
M \\
M \\
F \\
F \\
M \\
M \\
M \\
F \\
F \\
M \\
F \\
M \\
F \\
M \\
M \\
F \\
M \\
M \\
M \\
M \\
M \\
M \\
M \\
F \\
M \\
M \\
F \\
M \\
M \\
F \\
M \\
F \\
M \\
M \\
F \\
M\end{array}$ & $\begin{array}{l}75 \\
30 \\
70 \\
\text { Un } \\
75 \\
24 \\
28 \\
55 \\
9 \\
\text { Un } \\
76 \\
59 \\
55 \\
58 \\
45 \\
35 \\
57 \\
65 \\
40 \\
41 \\
25 \\
54 \\
18 \\
56 \\
28 \\
30 \\
31 \\
\text { Un } \\
59 \\
\text { Un } \\
\text { Un } \\
54 \\
60 \\
60 \\
37 \\
48 \\
40 \\
65\end{array}$ & $\begin{array}{l}C \\
C \\
C \\
C \\
C \\
C \\
C \\
C \\
C \\
C \\
C \\
C \\
C \\
P \\
P \\
P \\
P \\
P \\
P \\
\text { P } \\
C \\
C \\
C D \\
C D \\
C D \\
C D \\
C D \\
C D \\
C D\end{array}$ & $\begin{array}{l}\text { A } \\
\text { A } \\
\text { O } \\
\text { A } \\
\mathbf{N} \\
\mathbf{N} \\
\mathbf{N} \\
\mathbf{N} \\
\mathbf{N} \\
\mathbf{N} \\
\mathbf{N} \\
\mathbf{N} \\
\mathbf{N} \\
\mathbf{N} \\
\mathrm{U} \\
\mathrm{A} \\
\mathrm{A} \\
\mathbf{N} \\
\mathbf{N} \\
\mathbf{N} \\
\mathbf{N} \\
\mathbf{N} \\
\mathbf{N} \\
\mathbf{N} \\
\mathbf{N} \\
\mathrm{O} \\
\mathrm{O} \\
\mathbf{N} \\
\mathbf{N} \\
\mathbf{N} \\
\mathbf{N} \\
\mathbf{N} \\
\mathbf{A} \\
\mathrm{A} \\
\mathbf{U} \\
\mathrm{U} \\
\mathbf{N}\end{array}$ & $\begin{array}{l}39 / 17 \\
43 / 16 \\
20 / 17 \\
40 / 19 \\
21 / 18 \\
18 / 14 \\
28 / 15 \\
41 / 17 \\
23 / 17 \\
40 / 17 \\
23 / 21 \\
19 / 17 \\
39 / 18 \\
40 / 20 \\
41 / 16 \\
42 / 16 \\
40 / 17 \\
28 / 18 \\
21 / 15 \\
54 / 14 \\
42 / 14 \\
41 / 16 \\
17 / 17 \\
43 / 19 \\
16 / 15 \\
54 / 16 \\
49 / 16 \\
44 / 20 \\
41 / 23 \\
20 / 15 \\
19 / 18 \\
21 / 16 \\
38 / 16 \\
41 / 15 \\
46 / 16 \\
44 / 15 \\
41 / 16 \\
38 / 15\end{array}$ \\
\hline
\end{tabular}

Un = Unknown; $C=$ chorea; $P=$ psychiatric symptoms; $D=$ dementia; $R=$ rigidity; $A t=$ ataxia; $A=$ affected parent; $O=$ other affected relative; $N=$ no family history; $\mathrm{U}=$ uncertain

\section{Discussion}

In this series only $47 \%$ (seven of 15 ) of patients analysed who presented initially with chorea as their major manifestation had a normal length CAG repeat in the Huntington's disease gene. This is not surprising given that in six of these cases there was no evidence for Huntington's disease in preceding generations, but contrasts with the very high proportion $(98.4 \% \text { to } 100 \%)^{1011}$ of clinically established cases with a known family history that show the presence of the mutation. Ashizawa et $a l^{6}$ recently reported that five of $12(42 \%)$ patients thought clinically to have Huntington's disease but for whom no positive family history could be documented failed to demonstrate CAG expansions. The single mutation negative case (case 3) in our study where choreiform movements were the presenting feature and the patient had a positive family history for Huntington's disease merits some discussion. The affected relatives in this family did not include the patient's appropriate parent who had died at the age of 71 without any documented neurological dysfunction. The patient himself had been on antiparkinsonian medication before the onset of his chorea at the age of 70 . We did not have access to any DNA from other affected patients in this family that we could use to confirm the presence of the Huntington's disease mutation and therefore his clinical diagnosis of Huntington's disease remains insecure. We have previously reported a parent-child pair, clinically diagnosed as having Huntington's disease, both of whom were expansion negative (cases A and B, table 1, MacMillan et $\left.a l^{12}\right)$. Both have subsequently been found to have expansions in the CAG repeat sequence associated with dentatorubropallidoluysian atrophy (Ross et al, in preparation) emphasising that, wherever possible, even in the case of symptomatic patients said to have a "positive" family history, the mutation state of clinically affected family members should be ascertained.

Three of the six cases in this series presenting initially with psychiatric manifestations but with no family history of Huntington's disease were mutation positive. The first of these was a patient who presented with paranoid delusions at age 41 and developed a choreiform movement disorder 18 months later while taking phenothiazines. The involuntary movements were predominantly orofacial with lesser involvement of the upper limbs. There was no disturbance of eye movements, no evidence of cognitive decline, and his gait was normal. His mother was alive and well in her 70s and his father had died of a cardiac event in his 50 s. There had been no history of a movement disorder in his three sibs although one had died at the age of 26 . The second case was of a man who presented with a personality disorder at the age of 25 and subsequently developed progressive chorea (without a history of medication) from the age of 45 . His mother had died aged 92 and his father at the age of 70 , neither having 
displayed any features of Huntington's disease. His only sib was reported well at the age of 63 . The third case had presented with a psychiatric disturbance in her early $50 \mathrm{~s}$, which was shortly followed by chorea and dementia. Computed tomography showed only generalised cerebral atrophy. Her mother was alive and well at the age of 87 and her father had died aged 62 of malignancy. In all three cases the lack of a positive family history had made the referring clinicians suspicious of making a diagnosis of Huntington's disease although all developed a movement disorder (as had the three mutation negative patients) subsequent to the psychiatric presentation.

The results reported here are not directly comparable with those of Davis et al who examined a series of banked DNA samples from "isolated individuals with Huntington's disease" in addition to patients with clinically doubtful Huntington's disease. They defined a negative family history as both parents having died over 65 years of age without neurological or psychiatric disease or both parents still being alive. In our study the age at which parents had died was often not available. In four of our cases with negative family history it had been documented that both parents died over the age of 65 and without a history of a neurological disorder. In two of these cases an expanded repeat sequence was identified. It was not possible, due to the nature of the study, to exclude non-paternity as a reason for the negative family history. We think that a more likely explanation, however, is that these expansions ( 42 and 43 repeats) arose from "intermediate" alleles. ${ }^{13}$ Davis et $a \bar{P}$ found expanded repeat sequences in $89 \%$ of patients defined as clinically probable Huntington's disease and in $31 \%$ of those with clinically doubtful Huntington's disease. The referring clinicians in our study clearly thought that Huntington's disease was a possibility: we have not, however, attempted to subdivide the cases into "probable" or "doubtful" on the basis of the information provided. Benjamin et $a l^{\beta}$ reported mutation analysis results on 171 symptomatic patients, of which 56 were without a positive family history. Thirty eight $(70 \%)$ of these 56 had expansions of more than 36 repeats (Huntington's disease range $>36$ in their study). They found two symptomatic patients with repeat lengths in the normal range in families with mutation established Huntington's disease, a similar situation to that reported in our original series. ${ }^{12}$ Davis et $a \bar{l}$ defined a repeat length of less than 40 identified using the original primers as a borderline result and it is noteworthy that in all such cases repeat analysis with primers excluding the CCG region resulted in an increase in the recorded length of the CAG repeat. In our series we considered a CAG repeat length of 38 or greater to be disease related and did not find any values in the range 30-37. It is also our experience ( $P$ Davies, unpublished data) that allele lengths in the intermediate zone analysed with the original primer pair tend to enlarge when analysed with primers that omit the polymorphic CCG sequence.

This study, like those of Davis et $a l^{5}$ and Ashizawa et $a l,{ }^{6}$ shows how mutation analysis for the Huntington's disease CAG repeat expansion can be applied in clinical practice. It emphasises the need to be wary of a "negative" family history not only where there is limited knowledge of the status of "missing" parents but also where the parents have died at advanced age without neurological symptoms. The test undoubtedly is an important augmentation to the diagnosis of Huntington's disease in clinical practice. We would urge, as did Benjamin et $a l,{ }^{8}$ that it remains prudent to "proceed with care".

We thank the clinicians who forwarded samples for analysis and for their cooperation in completing the data forms and are grateful for the help of Gary Houlihan, nurse specialist and Joanna Solden, clinical psychologist, for their involvement with local patients. JCM is supported by an MRC Clinician Scientist Fellowship.

1 Harper PS. Huntington's disease. Philadelphia, WB Saunders, 1991

2 The Huntington's Disease Collaborative Research Group. A novel gene containing a trinucleotide repeat that is expanded and unstable on Huntington's disease chromosomes. Cell 1993;72:971-83.

3 Snell R, MacMillan JC, Cheadle JP, et al. Relationship between trinucleotide repeat expansion and phenotypic variation in Huntington's disease. Nature Genetics 1993; 4:393-7.

4 Andrew SE, Goldberg YP, Kremer B, et al. Huntington's disease without CAG expansion: phenocopies or errors in assignment? Am f Hum Genet 1994;54:852-63.

5 Davis MB, Bateman D, Quinn N, Marsden CD, Harding AE. Mutation analysis in patients with possible but AE. Mutation analysis in patients with possible but apparently

6 Ashizawa T, Wong L-J, Richards CS, Caskey CT, Janokovic J. CAG repeat size and clinical presentation in Huntington's disease. Neurology 1994;44:1137-43.

$7 \mathrm{St}$ Clair D. Expanded CAG trinucleotide repeat of Huntington's disease gene in a patient with schizophrenia and normal striatal histology. $f$ Med Genet 1994;31:658-60.

8 Benjamin CM, Adam S, Wiggins S, et al. Proceed with care: Direct predictive testing for Huntington's disease. Am 7 Med Genet 1994;55:606-17.

9 Tyler A, Ball D, Craufurd D. Presymptomatic testing for Huntington's disease in the United Kingdom. BMF 1992;304:1593-6.

10 Andrew SE, Goldberg YP, Kremer B, et al. The relationship between trinucleotide (CAG) repeat length and ship between trinucleotide (CAG) repeat length and
clinical features of Huntington's disease. Nature Genetics 1993;4:398-403.

11 Duyao M, Ambrose C, Myers R, et al. Trinucleotide repeat length instability and age of onset in Huntington's disease. Nature Genetics 1993;4:387-92.

12 MacMillan JC, Snell RG, Tyler A, et al. Molecular analyses and clinical correlations of the Huntington's disease mutation. Lancet 1993;342:954-8.

13 Goldberg YP, Kremer B, Andrew SE, et al. Molecular analysis of new mutations for Huntington's disease: intermediate alleles and sex of origin effects. Nature Genetics 1994;5:174-9. 